\title{
ON WEAKLY PRIME SUBMODULES
}

\author{
S. EBRAHIMI ATANI AND F. FARZALIPOUR
}

\|\|

\begin{abstract}
Let $R$ be a commutative ring with non-zero identity. We define a proper submodule $N$ of an $R$-module $M$ to be weakly prime if $0 \neq r m \in N(r \in R, m \in M)$ implies $m \in N$ or $r M \subseteq N$. A number of results concerning weakly prime submodules are given. For example, we give three other characterizations of weakly prime submodules.
\end{abstract}

\section{Introduction}

Throughout this paper $R$ will denote a commutative ring with non-zero identity and $M$ a unital module. Several authors have extended the notion of prime ideal to modules, see, for example [5, 6]. A proper ideal $P$ of $R$ is said to be weakly prime ideal if $0 \neq a b \in P$ implies $a \in P$ or $b \in P$. Weakly prime ideals in a commutative ring with non-zero identity have been introduced and studied by D. D. Anderson and E. Smith in [1]. Also, weakly primary ideals in a commutative ring with non-zero identity have been introduced and studied in [5]. Here we study weakly prime submodules of a module over a commutative ring. Various properties of weakly prime submodules are considered (see Sec. 2). In fact, the aim of this paper is to prove for weakly prime submodules some of the results given in [1] for weakly prime ideals. For example, we show that if $P$ is a submodule of a finitely generated multiplication $R$-module $M$, then $P$ is weakly prime if and only if for submodules $N$ and $K$ of $M$ with $0 \neq N K \subseteq P$, either $N \subseteq P$ or $K \subseteq P$.

Now we define the concepts that we will use. If $R$ is a ring and $N$ is a submodule of an $R$-module $M$, the ideal $\{r \in R: r M \subseteq N\}$ will be denoted by $(N: M)$. Then $(0: M)$ is the annihilator of $M$. An $R$-module $M$ is called a multiplication module if for each submodule $N$ of $M, N=I M$ for some ideal $I$ of $R$. In this case we can take $I=(N: M)($ see $[3])$.

Let $M$ be a module over a ring $R$. A proper submodule $N$ of $M$ with $(N: M)=P$ is said to be prime (or $P$-prime) if $r m \in N$ for $r \in R$ and $m \in M$ implies that either $m \in N$ or $r \in P$. We say that $M$ is a prime module if the zero submodule of $M$ is prime submodule of $M$ (see [ $[$ ) $)$.

Received October 21, 2005.

2000 Mathematics Subject Classification. Primary 13C05, 13C13, 13A15.

Key words and phrases. Weakly prime, multiplication, prime. 


\section{Weakly prime submodules}

Recall that a proper submodule $N$ of a module $M$ over a commutative ring $R$ is said to be weakly prime submodule if whenever $0 \neq r m \in N$, for some $r \in R, m \in M$, then $m \in N$ or $r M \subseteq N$. Clearly, every prime submodule of a module is a weakly prime submodule. However, since 0 is always weakly prime (by definition), a weakly prime submodule need not be prime.

Remark. We know that if $N$ is a prime submodule of an $R$-module $M$, then $(N: M)$ is a prime ideal of $R$. Suppose that $N$ is weakly prime which is not prime. Contrary to what happens for a prime submodules, the ideal $(N: M)$ is not, in general, a weakly prime ideal of $R$. For example, let $M$ denote the cyclic $Z$-module $Z / 8 Z$. Take $N=\{0\}$. Certainly $N$ is a weakly prime submodule of $M$, but $(N: M)=8 Z$ is not a weakly prime ideal of $R$, but we have the following results:

Proposition 2.1. Let $R$ be a commutative ring, $M$ a faithful cyclic $R$-module, and $N$ a weakly prime submodule of $M$. Then $(N: M)$ is a weakly prime ideal of $R$.

Proof. Assume that $M=R x$ and let $0 \neq a b \in(N: M)$ with $a \notin(N: M)$. Then there exists $r \in R$ such that $a(r x) \notin N$, so $a x \notin N$. As $0 \neq a b M \subseteq N$, it follows that $0 \neq a b x \in N$ (for if $a b x=0$, then $a b \in(0: x)=(0: M)=0$, a contradiction), so $b \in(N: M)$ since $N$ is a weakly prime submodule of $M$, as needed.

Proposition 2.2. Let $R$ be a commutative ring, $M$ a P-prime $R$-module, and $N$ a weakly prime submodule of $M$. Then $(N: M)$ is a weakly prime ideal of $R$.

Proof. Let $0 \neq a b \in(N: M)$ with $a \notin(N: M)$. Then there exists $m \in M-N$ such that $a m \notin N$. As $0 \neq a b M \subseteq N$ we have $a b m \in N$. If $a b m=0$, then $a b \in(0: m)=(0:$ $M)=P$, so $b \in P \subseteq(N: M)$. If $a b m \neq 0$, then $b \in(N: M)$ since $a m \notin N$ and $N$ is a weakly prime submodule of $M$, as required.

If we adapt the proof of the [4, Proposition 2.14 and Theorem 2.15], then we get the following corollary:

Corollary 2.3. Let $R$ a commutative ring, $M$ an $R$-module, and $N$ a weakly prime submodule of $M$ that is not prime. Then the following hold:

(i) If $I$ is an ideal of $R$ such that $I \subseteq(N: M)$, then $I N=0$. In particular, $(N$ : $M) N=0$.

(ii) If $M$ is a multiplication $R$-module, then $N^{2}=0$.

We next give three other characterizations of weakly prime submodules.

Theorem 2.4. Let $R$ be a commutative ring, $M$ an $R$-module, and $N$ a proper submodule of $M$. Then the following statements are equivalent.

(i) For ideal $I$ of $R$ and submodule $D$ of $M$ with $0 \neq I D \subseteq N$, either $I M \subseteq N$ or $D \subseteq N$. 
(ii) $N$ is a weakly prime submodule of $M$.

(iii) For $m \in M-N,(N: R m)=(N: M) \cup(0: R m)$.

(iv) For $m \in M-N,(N: R m)=(N: M)$ or $(0: R m)=(N: R m)$.

Proof. (i) $\Rightarrow$ (ii) Suppose that $0 \neq s m \in N$ where $s \in R$ and $m \in M$. Take $I=R s$ and $D=R m$. Then $0 \neq I D \subseteq N$, so either $I \subseteq(N: M)$ or $D \subseteq N$; hence either $r \in(N: M)$ or $m \in N$. Thus $N$ is weakly prime.

(ii) $\Rightarrow$ (i) Suppose that $N$ is a weakly prime submodule of $M$. If $N$ is prime, then the result is clear. So we can assume that $N$ is weakly prime that is not prime. Let $0 \neq I D \subseteq N$ with $x \in D-N$. We show that $I \subseteq(N: M)$. Let $r \in I$. If $0 \neq r x$, then $N$ weakly prime gives $r \in(N: M)$. So assume that $r x=0$. First suppose that $r D \neq 0$, say $r d \neq 0$ where $d \in D$. If $d \notin N$, then $r \in(N: M)$. If $d \in N$, then $r(d+x)=r d \in N$, so $r \in(N: M)$ or $d+x \in N$. Thus, $r \in(N: M)$; hence $I \subseteq(N: M)$. So we can assume that $r D=0$. Suppose that $I x \neq 0$, say $a x \neq 0$ where $a \in I$. Then $N$ weakly prime gives $a \in(N: M)$. As $(r+a) x=a x \in N$, we get $r \in(N: M)$, so $I \subseteq(N: M)$. Therefore, we can assume that $I x=0$.

Since $I D \neq 0$, there exist $b \in I$ and $d_{1} \in D$ such that $b d_{1} \neq 0$. As $(N: M) N=0$ (by Corollary 2.3.) and $0 \neq b\left(d_{1}+x\right)=b d_{1} \in N$ we can divide the proof into the following two cases:

Case 1. $b \in(N: M)$ and $d_{1}+x \notin N$.

Since $0 \neq(r+b)\left(d_{1}+x\right)=b d_{1} \in N$, we obtain $r+b \in(N: M)$, so $r \in(N: M)$. Hence $I \in(N: M)$.

Case 2. $b \notin(N: M)$ and $d_{1}+x \in N$.

As $0 \neq b d_{1} \in N$ we have $d_{1} \in N$, so $x \in N$ which is a contradiction. Thus $I \subseteq(N$ : $M)$.

(ii) $\Rightarrow$ (iii) Clearly, if $m \in M-N$, then $H=(N: M) \cup(0: R m) \subseteq(N: R m)$. Let $a \in(N: R m)$ where $m \in M-N$. Then $a m \in N$. If $a m \neq 0$, then $a \in(N: M)$ since $N$ is weakly prime, so $a \in H$. If $a m=0$, then $a \in(0: R m)$, so $a \in H$, and hence we have equality.

(iii) $\Rightarrow$ (iv) Is obvious.

(iv) $\Rightarrow$ (ii) Suppose that $0 \neq r m \in N$ with $r \in R$ and $m \in M-N$. Then $r \in(N: R m)$ and $r \notin(0: R m)$. It follows from (iv) that $r \in(N: R m)=(N: M)$, as required.

Let $M$ be an $R$-module and $N$ be a submodule of $M$ such that $N=I M$ for some ideal $I$ of $R$. Then we say that $I$ is a presentation ideal of $N$. Note that it is possible that for a submodule $N$, no such presentation ideal exist. For example, assume that $M$ is a vector space over an arbitrary field $F$ with $\operatorname{dim}_{F} M \geq 2$ and let $N$ be a proper subspace of $M$ such that $N \neq 0$. Then $M$ is finite length (so $M$ is noetherian, artinian and injective), but $M$ is not multiplication and $N$ has not any presentation. Clearly, every submodule of $M$ has a presentation ideal if and only if $M$ is a multiplication module.

Let $N$ and $K$ be submodules of a multiplication $R$-module $M$ with $N=I_{1} M$ and $K=I_{2} M$ for some ideals $I_{1}$ and $I_{2}$ of $R$. The product $N$ and $K$ denoted by $N K$ is defined by $N K=I_{1} I_{2} M$. Then by [2, Theorem 3.4], the product of $N$ and $K$ is 
independent of presentations of $N$ and $K$. Moreover, for $a, b \in M$, by $a b$, we mean the product of $R a$ and $R b$. Clearly, $N K$ is a submodule of $M$ and $N K \subseteq N \cap K$ (see [2]).

Lemma 2.5. Let $M$ be a multiplication module over a commutative ring $R$, and let $N$ and $K$ be submodules of $M$.

(i) If for every $a \in N$, aK=0, then $N K=0$.

(ii) If for every $b \in K, N b=0$, then $N K=0$.

(iii) If $a b=0$ for every $a \in N, b \in K$, then $N K=0$.

Proof. (i) Assume that $a \in N$ and let $I_{a}$ and $J$ be the presentation ideals $R a$ and $K$ respectively. Then $a K=(R a) K=I_{a} J M=0$, so $I_{a} J \subseteq(0: M)$. By [2, Proposition 3.8] we have

$$
N K=\left(\sum_{a \in N} R a\right) K=\left(\sum_{a \in N} I_{a} M\right)(J M)=\left(\sum_{a \in N} I_{a} J\right) M \subseteq(0: M) M=0 .
$$

(ii) This proof is similar to that in case (i) and we omit it.

(iii) Assume that $a \in N, b \in K$. There are ideals $I_{a}$ and $J_{b}$ of $R$ such that $a b=$ $(R a)(R b)=I_{a} J_{b} M=0$, so $I_{a} J_{b} \subseteq(0: M)$. By [2, Proposition 3.8] we have

$$
N K=\left(\sum_{a \in N} R a\right)\left(\sum_{b \in K} R b\right)=\left(\sum_{a \in N} \sum_{b \in K} I_{a} J_{b}\right) M \subseteq(0: M) M=0 .
$$

Compare the next result with [1, Theorem 3(4)].

Theorem 2.6. Let $R$ be a commutative ring, $M$ a finitely generated multiplication $R$-module, and $P$ a proper submodule of $M$. Then the following statements are equivalent.

(i) $P$ is a weakly prime submodule of $M$.

(ii) For submodules $N$ and $K$ of $M$ with $0 \neq N K \subseteq P$, either $N \subseteq P$ or $K \subseteq P$.

Proof. (i) $\Rightarrow$ (ii) If $P$ is prime, then (ii) follows from [2, Theorem 3.16], so we can assume that $P$ is not prime. Suppose that $N$ and $K$ are submodules of $M$ with $0 \neq$ $N K \subseteq P$, but $N \nsubseteq P$ and $K \nsubseteq P$. We show that $N K=0$ which is a contradiction. Let $a \in N$. First, suppose that $a \in N-P$. Let $I_{1}, I_{2}$ and $I_{3}$ be the presentation ideals $R a$, $N$ and $K$, respectively. Since $R a \subseteq N$ and $N K \subseteq P$, we have $I_{1} \subseteq I_{2}+(0: M)=I_{2}$ and $I_{2} I_{3} \subseteq(P: M)$ by $\left[7\right.$, p.231 Corollary], so $a \bar{K}=(R a) K=I_{1} I_{3} M \subseteq I_{2} I_{3} M \subseteq(P$ : $M) M=P$; hence $I_{3} \subseteq(P: R a)$. It follows from Theorem 2.4 that either $I_{3} \subseteq(P: N)$ or $I_{3} \subseteq(0: R a)$. As $K \nsubseteq P$ we have $I_{3} \subseteq(0: R a)$. Therefore, $a K=(R a) I_{3} M=0$, so $N K=0$ by Lemma 2.5. Next suppose that $a \in N \cap P$. Let $b \in K$. If $b \in P$, then $a b=(R a)(R b) \subseteq P^{2}=0$ by Corollary 2.3 , so $N K=0$. If $b \in K-P$, then as previously noted, $N b=0$, hence $a b \subseteq N b=0$, so $N K=0$ by Lemma 2.5 .

(ii) $\Rightarrow$ (i) Suppose that $0 \neq I D \subseteq N$ where $I$ is an ideal of $R$ and $D$ is a submodule of $M$. Take $K=I M$. Then $0 \neq K D=I(D: M) M=I D \subseteq N$, so either $K=I M \subseteq(N$ : $M) M=N$ or $D \subseteq N$ by (ii). It follows from [8, p.231 Corollary] that either $I \subseteq(N: M)$ or $D \subseteq N$. Thus $N$ is weakly prime by Theorem 2.5 . 
Let $N$ be a proper submodule $M$. Then, the radical of $N$ denoted by $\sqrt{N}$ is defined in [3] to be the intersection of all prime submodules of $M$ containing $N$. If $N$ is a submodule of a multiplication $R$-module, then by [2, Theorem 3.13] we have

$$
\sqrt{N}=\left\{m \in M: m^{k} \subseteq N \text { for some non-zero integer } k\right\}
$$

Compare the following theorem with [1, Theorem 4].

Theorem 2.7. Let $R$ be a commutative ring, $M$ a finitely generated faithful multiplication $R$-module, and $N$ a weakly prime submodule of $M$ that is not prime. Then $N \sqrt{0}=0$.

Proof. Let $x \in \sqrt{0}$. Then $R x=J M$ and $N=I M$ for some ideals $J$ and $I$ of $R$. If $x \in N$, then $J \subseteq I$ by [7, p.231 Corollary] and $N(R x)=I J M \subseteq N^{2}=0$ by Corollary 2.3 , so $N x=0$. It follows from Lemma 2.5 that $N \sqrt{0}=0$. So suppose that $x \notin N$. By Theorem 2.4, either $(N: R x)=(N: M)$ or $(N: R x)=(0: R x)$. If $(N: R x)=(0: R x)$, then $N \subseteq(N: R x) M=(0: R x) M$ (since $(N: M) \subseteq(N: R x))$. Therefore, $N_{x} \subseteq J(0:$ $R x) M=0$, and hence $N \sqrt{0}=0$ by Lemma 2.5. Suppose that $(N: M)=(N: R x)$. Let $x^{n}=J^{n} M=0$, but $x^{n-1}=J^{n-1} M \neq 0$, so $J^{n}=0$ by [7, p.231 Corollary]. As $J^{n-1} \subseteq(I: J)$ we have $0 \neq x^{n-1} \subseteq(I M, J M) M=(N: R x) M=(N: M) M=N$, so $x \in N$ by Theorem 2.6 which is a contradiction. Thus $N \sqrt{0}=0$.

Corollary 2.8. Let $M$ be a finitely generated faithful multiplication module over a commutative ring $R$. Suppose that $N$ and $K$ are weakly prime submodules of $M$ that are not prime. Then $N K=0$.

Proof. Assume that $N$ and $K$ are weakly prime submodule of $M$ that are not prime. If $b \in N$, then $b^{2} \in N^{2}=0$ by Corollary 2.3 , so $N \subseteq \sqrt{0}$. Similarly, $K \subseteq \sqrt{0}$. Then $N K \subseteq N \sqrt{0}=0$ by Theorem 2.7, as required.

Corollary 2.9. Let $M$ be a finitely generated faithful multiplication module over a commutative ring $R$ with unique maximal submodule $N$, and let every prime submodule of $M$ is maximal. If $P$ is a weakly prime submodule of $M$, then either $P=N$ or $P N=0$.

Proof. If $P$ is prime, then $P=N$. If $P$ is not prime, then $\sqrt{0}=N$ and $P N=0$ by Theorem 2.7.

Corollary 2.10. Let $M$ be a finitely generated faithful module over a local ring $R$ with unique maximal submodule $N$, and let every prime submodule of $M$ is maximal. If $P$ is a weakly prime submodule $M$, then either $P=N$ or $P N=0$.

Proof. By [6, p.3748 Corollary 1], $M$ is cyclic, and hence $M$ is a multiplication $R$-module. Now the asserition follows from Corollary 2.9. 


\section{References}

[1] D. D. Anderson and E. Smith, Weakly prime ideals. Houston J. of Math. 29(2003), 831-840.

[2] R. Ameri, On the prime submodules of multiplication modules. Inter. J. of Math. and Mathematical Sciences 27(2003), 1715-1724.

[3] Z. A. El-Bast and P. F. Smith, Multiplication modules. Comm. in Algebra 16(1988), 755779 .

[4] S. Ebrahimi Atani, The product of multiplication submodules. Honam Mathematical Journal 27(2005), 1-8.

[5] J. Dauns, Prime modules, J. reine Angew. Math. 2(1978), 156-181.

[6] C. P. Lu, Spectra of modules. Comm. in Algebra 23(1995), 3741-3752.

[7] P. F. Smith, Some remarks on multiplication modules, Arch. Math. 50(1988), 223-235.

Department of Mathematics, University of Guilan P. O. Box 1914 Rasht Iran.

E-mail: ebrahimi@guilan.ac.ir 